\title{
Evolución del contenido de ácidos grasos de aceite de aguacate durante la maduración
}

\author{
Por L. Martínez Nieto y M.a V. Moreno Romero
}

Dpto. Ingeniería Química. Facultad de Ciencias. Universidad de Jaén. Campus Universitario Las Lagunillas. 23071 JAEN, y

Grupo de Investigación de Biorreactores. Dpto. Ingeniería Química. Facultad de Ciencias. Universidad de Granada. Campus Universitario Fuente Nueva. 18071 GRANADA.

RESUMEN

Evolución del contenido de ácidos grasos de aceite de aguacate durante la maduración.

La riqueza grasa mínima exigida al aguacate para su salida al mercado es del $8 \%$, aunque en la maduración vegetativa se alcanzan valores de alrededor del $20 \%$ o más según variedades. En el período que transcurre desde el corte del fruto, de acuerdo con la madurez comercial, hasta la completa madurez del mismo no sólo aumenta la riqueza grasa, sino que se ha observado una evolución del contenido graso, de forma que hay un aumento de los ácidos grasos insaturados en detrimento del contenido en ácidos grasos saturados.

PALABRAS-CLAVE: Aceite de aguacate - Acido graso (contenido en) - Aguacate - Maduración (influencia de).

\section{SUMMARY}

Changes in the fatty acids of avocado oil during maturation.

The minimum fat richness of avocados necessary for marketing of the fruit is $8 \%$, although after vegetative maturation values of about $20 \%$ or more are reached, the values depending on the variety. In the period between harvesting, when commercial maturity is reached, and full maturation, not only is the fat content increased but changes also occur in the fat composition, the unsaturated fatty acids increasing while the amount of saturated fatty acids decreases.

KEY-WORDS: Avocado - Avocado oil - Fatty acid (content in) Ripening (influence of).

\section{INTRODUCCION}

El cultivo de aguacate ha adquirido gran importancia en las provincias de Granada y Málaga, donde se produce intensivamente, aunque sea susceptible de cultivarse en otras zonas mediterráneas o atlánticas. Su producción se estima podrá alcanzar del orden de las $100.000 \mathrm{Tm}$ para los próximos años, de los que aproximadamente un $60 \%$ se exportarán y el resto se destinarán a consumo interior si sigue la tendencia actual (1).

El valor alimenticio e industrial del aguacate se debe a su gran riqueza en materia grasa, lo que le da un lugar relevante entre las diferentes categorías de frutos.

El fruto español se destina fundamentalmente a consumo alimentario, habiendo estado controlada por organismos oficiales la madurez necesaria para su salida al mercado, exigiéndose una riqueza mínima del $8 \%(2)$.
Por su composición química se encuentra entre los frutos más ricos en materia grasa y no puede ser comparado desde este punto de vista más que con la aceituna. La riqueza grasa media del aguacate maduro oscila alrededor del $20 \%$.

El aceite extraído de la pulpa de aguacate tiene una consistencia espesa, un color verde intenso y un sabor agradable. Puede considerarse formado por ácidos grasos, vitaminas y materia insaponificable. En función de la variedad y la composición del aguacate aparece un elevado porcentaje de ácidos grasos poliinsaturados, beneficiosos para la salud. Por ello, parece importante conocer la evolución de dichos ácidos grasos con la maduración vegetativa con objeto de establecer el período de corte no tanto en función del mínimo contenido graso exigido sino en función del máximo contenido en ácidos grasos poliinsaturados (3), (4), (5).

En el presente trabajo se da cuenta de los resultados obtenidos del análisis de la composición del aceite de aguacate de varias cosechas y distintas variedades en función de la fecha de corte.

\section{MATERIALES Y METODOS}

Los aguacates empleados procedían de Torrecuevas, El Puntalón y Pataura (Motril), Salobreña así como Benamargosa y Coín (Málaga). De cada muestra, se conocía tanto la zona de procedencia como la fecha de corte.

Una vez el fruto en nuestro poder se procedía inmediatamente a la división del mismo en dos calibres. El primero denominado "alto" formado por frutos de más de $225 \mathrm{~g}$ (calibre comercial 16-14 o inferiores) y el segundo denominado "bajo" formado por frutos de menos de $225 \mathrm{~g}$ (calibre comercial 18-20 o superiores). Posteriormente se efectúa la toma de muestras, que consiste en tomar tres frutos de cada calibre y variedad y dos muestras por fruto.

A continuación se realiza el secado y la extracción para lo cual se parte el aguacate en sentido meridional, para eliminar el hueso, se corta en láminas de $2 \mathrm{~mm}$ de espesor y se seca en el horno microondas hasta pesada constante (aproximadamente $15 \mathrm{~min}$ ) (2).

Una vez seca la pulpa, se coloca en un cartucho de papel de filtro y se realiza la extracción en soxhlet, utili- 
zando hexano como disolvente. El tiempo de extracción es de 4 horas (6), (7), (8).

El extracto, colocado en un matraz seco y previamente tarado, se somete a evaporación en un evaporador rotatorio a vacío para eliminar el disolvente.

El aceite obtenido se seca en estufa de vacío a $60^{\circ} \mathrm{C}$ y $20 \mathrm{~mm} \mathrm{Hg}$. Posteriormente, se mantiene en un desecador durante una hora aproximadamente y se procede a su pesada.

Para la realización del análisis de la composición del aceite en ácidos grasos se ha seguido fundamentalmente la Norma U.N.E. 55037 (9), (10). Se ha utilizado un cromatógrafo Carlo Erba equipado con una columna de acero inoxidable de Chromosorb W AW 80-100 con 10\% de D.E.G.S., en las siguientes condiciones de trabajo: temperatura del horno $170^{\circ} \mathrm{C}$; temperatura de inyección $210^{\circ} \mathrm{C}$; temperatura del detector $200^{\circ} \mathrm{C}$; flujo de gas portador $\left(\mathrm{N}_{2}\right) 15 \mathrm{ml} / \mathrm{min}$.

\section{RESULTADOS Y DISCUSION}

En las Tablas I, II y III se han recogido los valores de la composición en ácidos grasos individuales correspondientes a las variedades. "Bacon" "Fuerte" y "Hass", que cubren aproximadamente el $95 \%$ de la cuota de mercado, según zonas de producción y distintas fechas de corte en la época comercial de cada variedad. Cada valor es media de 3 muestras.

Tabla I

Composición en ácidos grasos del aceite de aguacate durante la maduración variedad "Bacon"

\begin{tabular}{ccccccccccc}
\hline Año & Zona & Fecha corte & Calibre & C16:0 & C16:1 & C18:0 & C18:1 & C18:2 & C18:3 & N.I. \\
\hline 1989 & Puntalón & 1-Octubre & Bajo & 17,9 & 6,8 & - & 57,8 & 15,2 & 1,3 & 1,0 \\
1989 & Puntalón & 16-Octubre & Bajo & 17,2 & 10,4 & 0,6 & 58,6 & 12,4 & 0,8 & - \\
1989 & Puntalón & 1-Octubre & Alto & 16,9 & 8,6 & - & 55,6 & 17,4 & 1,4 & 0,1 \\
1989 & Puntalón & 16-Octubre & Alto & 15,2 & 8,7 & 0,7 & 62,7 & 12,0 & 0,9 & - \\
1989 & Torrecuevas & 24-Octubre & Bajo & 15,0 & 7,5 & 0,6 & 60,4 & 15,3 & 1,1 & 0,1 \\
1989 & Torrecuevas & 4-Noviembre & Bajo & 16,0 & 7,2 & - & 63,2 & 12,7 & 0,9 & - \\
1989 & Torrecuevas & 24-Octubre & Alto & 15,7 & 10,6 & 0,7 & 59,3 & 12,8 & 0,9 & - \\
1989 & Torrecuevas & 4-Noviembre & Alto & 14,6 & 7,9 & 0,5 & 64,1 & 11,8 & 1,1 & - \\
1990 & Pataura & 8-Octubre & Alto & 19,9 & 8,7 & 0,7 & 55,4 & 12,6 & 0,7 & 1,9 \\
1990 & Pataura & 23-Octubre & Alto & 17,2 & 8,1 & 0,6 & 59,0 & 13,0 & 0,7 & 0,6 \\
1990 & Pataura & 5-Noviembre & Alto & 15,1 & 8,2 & 0,5 & 64,3 & 10,7 & 0,7 & 0,5 \\
1990 & Salobreña & 11-Noviembre & Bajo & 17,5 & 7,8 & 0,6 & 61,6 & 11,5 & 0,7 & 0,4 \\
1990 & Salobreña & 22-Noviembre & Bajo & 14,9 & 5,9 & 0,7 & 66,9 & 10,9 & 0,7 & - \\
1990 & Salobreña & 11-Noviembre & Alto & 19,7 & 9,8 & 0,6 & 59,5 & 9,8 & 0,6 & - \\
1990 & Salobreña & 22-Noviembre & Alto & 17,1 & 8,3 & 0,6 & 64,1 & 9,9 & - & - \\
\hline
\end{tabular}

Tabla II

Composición en ácidos grasos del aceite de aguacate durante la maduración variedad "Fuerte"

\begin{tabular}{ccccccccccc}
\hline Año & Zona & Fecha corte & Calibre & C16:0 & C16:1 & C18:0 & C18:1 & C18:2 & C18:3 & N.I. \\
\hline 1989 & Benamargosa & 13-Noviembre & Bajo & 17,9 & 6,8 & - & 57,8 & 15,2 & 1,3 & 1,0 \\
1990 & Benamargosa & 4-Febrero & Bajo & 17,2 & 10,4 & 0,6 & 58,6 & 12,4 & 0,8 & - \\
1989 & Benamargosa & 13-Noviembre & Alto & 16,9 & 8,6 & - & 55,6 & 17,4 & 1,4 & 0,1 \\
1990 & Benamargosa & 4-Febrero & Alto & 15,2 & 8,7 & 0,7 & 62,7 & 12,0 & 0,9 & - \\
1991 & Benamargosa & 15-Enero & Bajo & 15,0 & 7,5 & 0,6 & 60,4 & 15,3 & 1,1 & 0,1 \\
1991 & Benamargosa & 4-Febrero & Bajo & 16,0 & 7,2 & - & 63,2 & 12,7 & 0,9 & - \\
1991 & Benamargosa & 15-Enero & Alto & 15,7 & 10,6 & 0,7 & 59,3 & 12,8 & 0,9 & - \\
1991 & Benamargosa & 4-Febrero & Alto & 14,6 & 7,9 & 0,5 & 64,1 & 11,8 & 1,1 & - \\
\hline
\end{tabular}


Tabla III

Composición en ácidos grasos del aceite de aguacate durante la maduración variedad "Hass"

\begin{tabular}{ccccccccccc}
\hline Año & Zona & Fecha corte & Calibre & C16:0 & C16:1 & C18:0 & C18:1 & C18:2 & C18:3 & N.I. \\
\hline 1990 & Pataura & 7-Febrero & Bajo & 18,0 & 10,8 & 0,4 & 56,0 & 13,7 & 0,8 & 0,3 \\
1990 & Pataura & 11-Marzo & Bajo & 18,5 & 9,1 & 0,4 & 59,1 & 11,5 & 0,7 & 0,6 \\
1990 & Pataura & 7-Febrero & Alto & 17,2 & 8,1 & 0,6 & 59,0 & 13,1 & 0,7 & 0,5 \\
1990 & Pataura & 11-Marzo & Alto & 15,1 & 8,2 & 0,5 & 64,3 & 10,8 & 0,6 & 0,5 \\
1991 & Pataura & 14-Enero & Bajo & 15,8 & 9,5 & 0,4 & 62,5 & 10,6 & 0,8 & 0,4 \\
1991 & Pataura & 21-Enero & Bajo & 14,4 & 8,8 & 0,4 & 65,8 & 9,5 & 0,7 & 0,4 \\
1991 & Pataura & 14-Enero & Alto & 16,5 & 8,6 & 0,4 & 64,2 & 9,1 & 0,5 & 0,7 \\
1991 & Pataura & 21-Enero & Alto & 13,4 & 7,9 & 0,4 & 67,8 & 9,6 & 0,7 & 0,2 \\
1992 & Coín & 14-Enero & Bajo & 17,7 & 9,1 & 0,5 & 60,7 & 11,1 & 0,6 & 0,3 \\
1992 & Coín & 3-Febrero & Bajo & 15,1 & 7,0 & 0,5 & 64,3 & 11,9 & 0,9 & 0,3 \\
1992 & Coín & 9-Marzo & Bajo & 13,9 & 6,1 & 0,5 & 69,5 & 9,1 & 0,6 & 0,3 \\
1992 & Coín & 14-Enero & Alto & 17,3 & 8,5 & 0,4 & 63,0 & 9,8 & 0,6 & 0,4 \\
1992 & Coín & 3-Febrero & Alto & 18,4 & 10,4 & 0,5 & 56,0 & 13,6 & 0,8 & 0,3 \\
1992 & Coín & 9-Marzo & Alto & 14,2 & 6,2 & 0,4 & 68,3 & 10,1 & 0,8 & 0,2 \\
\hline
\end{tabular}

De la observación de los resultados recogidos en estas tablas sólo se aprecia que el retraso de la cosecha del fruto implica un claro incremento del nivel de ácido oleico (C18:1), y una disminución del contenido de ácido linoleico (C18:2). El nivel de esteárico (C18:0) permanece constante, y tanto palmítico (C16:0) como palmitoleico (C16:1) presentan una cierta tendencia a la baja.
Para poder comparar mejor la evolución del contenido global de ácidos grasos se ha efectuado para cada una de las muestras ensayadas la suma de ácidos grasos insaturados; por una parte, y la de los saturados, por otra, y se ha efectuado el cociente entre ambas. En las Tablas IV, V y VI se encuentran los resultados de dichos cálculos para cada una de las zonas de producción, variedades, calibres y cosechas.

Tabla IV

Evolución del contenido en ácidos grasos del aceite de aguacate durante la maduración variedad "Bacon"

\begin{tabular}{lcccc}
\hline Año & Zona & Período & Calibre & $\Sigma$ A. Grasos insaturados / $\Sigma$ A. Grasos saturados \\
\hline 1989 & Puntalón & $1 / X-16 / X$ & Bajo & $4,53-4,62$ \\
1989 & Puntalón & $1 / X-16 / X$ & Alto & $4,91-5,30$ \\
1989 & Torrecuevas & $24 / X-4 / X I$ & Bajo & $5,25-5,40$ \\
1989 & Torrecuevas & $23 / X-4 / X I$ & Alto & $5,09-5,62$ \\
1990 & Pataura & $8 / X-5 / X I$ & Alto & $3,76-5,38$ \\
1990 & Salobreña & $11 / X I-22 / X I$ & Bajo & $4,52-5,41$ \\
1990 & Salobreña & $11 / X I-22 / X I$ & Alto & $3,93-4,65$ \\
\hline
\end{tabular}

Tabla V

Evolución del contenido en ácidos grasos del aceite de aguacate durante la maduración variedad "Fuerte"

\begin{tabular}{ccccc}
\hline Año & Zona & Período & Calibre & $\Sigma$ A. Grasos insaturados / $\Sigma$ A. Grasos saturados \\
\hline 1989 & Benamargosa & $13 / X \mid-4 / I$ & Bajo & $4,99-5,24$ \\
1989 & Benamargosa & $13 / X I-4 / I I$ & Alto & $4,99-5,25$ \\
1990 & Benamargosa & $15 / /-4 / I I$ & Bajo & $4,47-4,63$ \\
1990 & Benamargosa & $15 / I-4 / I I$ & Alto & $4,45-4,67$ \\
\hline
\end{tabular}


Tabla VI

Evolución del contenido en ácidos grasos del aceite de aguacate durante la maduración variedad "Hass"

\begin{tabular}{ccccc}
\hline Año & Zona & Período & Calibre & $\Sigma$ A. Grasos insaturados / $\Sigma$ A. Grasos saturados \\
\hline 1990 & Pataura & $7 / I I-11 / I I I$ & Bajo & $4,25-4,42$ \\
1990 & Pataura & $7 / I I-11 / I I I$ & Alto & $4,54-5,38$ \\
1991 & Pataura & $14 / I-26 / I I$ & Bajo & $5,17-5,73$ \\
1991 & Pataura & $14 / I-26 / I I$ & Alto & $4,88-6,23$ \\
1992 & Coín & $14 / I-9 / I I I$ & Bajo & $4,48-7,17$ \\
1992 & Coín & $14 / I-9 / I I I$ & Alto & $4,62-5,83$ \\
\hline
\end{tabular}

De la observación de dichas tablas se deduce que el cociente $\Sigma$ ácidos grasos insaturados $/ \Sigma$ ácidos grasos saturados aumenta en todos los casos, pudiendo apreciarse que este incremento es más ostensible cuando el período transcurrido entre la primera y la última corta es mayor.

\section{CONCLUSIONES}

De lo anteriormente expuesto se puede concluir que, con independencia de la zona productiva, variedad y calibre ensayados, si bien el aguacate cortado con el mínimo de riqueza grasa exigible puede tener unos caracteres organolépticos suficientes para su salida al mercado, desde el punto de vista nutricional es conveniente dejar el fruto en el árbol el mayor tiempo posible, ya que de esa forma la composición de su grasa aumenta en ácidos grasos insaturados que son beneficiosos para la salud. De lo expuesto se deduce que esta condición se cumplirá a medida que la fruta esté más próxima a la maduración climatérica y no a la comercial.

\section{BIBLIOGRAFIA}

1. S.O.I.V.R.E., Granada (1991).

2. Young, R.E. (1981).- "California Avocado Comission".- Universidad de California. Riverside, 92521.

3. Mataix Verdú, F.J. y Martínez de Victoria Muñoz, E. (1988).- "El Aceite de Oliva. Bases para el Futuro".- Junta de Andalucía. Consejería de Agricultura y Pesca.

4. Martínez de Victoria, E y Mañas, M. (1985) - "Estado Actual del Conocimiento del Valor Nutricional del Aceite de Oliva".- Ponencia presentada al II Simposium Cientifico del Aceite de Oliva. Jaén.

5. Mataix Verdú, F.J. (1985).- "Visión Actual de la Influencia del Consumo de Aceites Poliinsaturados en la Salud del Hombre".- Ponencia presentada al II Simposium Científico del Aceite de Oliva. Jaén.

6. Sadir, R. (1972).- "Olio di Avacate: Tecnologia dell'Estrazione e Industrializzazione dei Residui".- Riv. Ital. Sostanze Grasse 49, 90-93.

7. Wolff, J.P. (1954).- "Etude Spectrophotométrique du Rancissement des Huiles".- Rev. Fr. Corps Gras 1, 214-226.

8. Alter, M. y Gutfinger, T. (1982).- "Phospholipids in Several Vegetable Oils".- Riv. Ital. Sostanze Grasse 59, 14-18.

9. Norma U.N.E. 55037.

10. "Metodos de Análisis de Aceites y Grasas". Ministerio de Agricultura, n. 41.

Recibido: Septiembre 1994

Aceptado: Marzo 1995 